DOI:10.2478/rrlm-2021-0035

\title{
Mutations in the $K R A S$ gene as a predictive biomarker of therapeutic response in patients with colorectal cancer
}

\author{
Dragana Jugovićc ${ }^{*}$, Marija Vukelić Nikolić², Višnja Madić ${ }^{3}$ Ljiljana Branković ${ }^{1}$, \\ Radovan Milićević ${ }^{1}$, Goran Stanojević ${ }^{4}$, Perica Vasiljević ${ }^{3}$
}
1. Laboratory for Immunology and Genetics, Center for Medical and Clinical biochemistry, University Clinical Center Niš, Serbia
2. Faculty of Medicine, Research Center for Biomedicine, University of Niš, Serbia

3. Department of Biology and Ecology, Faculty of Science and Mathematics, University of Niš, Serbia

4. Clinic for digestive surgery, University Clinical Center Niš, Serbia

\begin{abstract}
Introduction: Despite the important role of general KRAS mutational status in the selection of an adequate therapeutic protocol in patients with colorectal cancer (CRC), studies that focus on its specific mutations and their significance on progression of disease are scarce. This study aimed to determine the significance of specific KRAS mutations in response to standard chemotherapy protocols with oxaliplatin-based (FOLFOX 4, OXFL) in the firstline and irinotecan-based chemotherapy (FOLFIRI, IFL) in the second-line therapy, and to evaluate the correlation between these mutations and clinicopathological characteristics of CRC patients. Methods: Genomic DNA was extracted from the FFPE tumour tissue sections while the KRAS mutation test was performed by using PCR methods. Results: Prevalence of KRAS gene mutations in CRC patients was $45 \%$. Mutated KRAS was more frequent in later stages of tumor infiltrations $(P=0.0017)$, on the right side of the colon $(P=0.0044)$, and in patients who developed metastases in the first 6 months after CRC diagnosis than in patients who developed metastases after 24 months $(P=0.0083)$. In a group of patients with a poor therapeutic response to standard chemotherapy the most frequent mutations in KRAS gene were G12D and G12V (63.88\%), while in a group of patients with a good response to therapeutic protocols the most prevalent mutation was G12A (66.66\%). Conclusion: Our results indicate that there was a significant difference in biological behaviour between tumours harboring different mutations in KRAS gene. Overall, mutation G12A could be a novel prognostic biomarker for CRC patients treated with standard chemotherapy.
\end{abstract}

Keywords: colorectal cancer, biomarker, real-time PCR, KRAS gene

Received: $6^{\text {th }}$ October 2021; Accepted: 11 $1^{\text {th }}$ October 2021; Published: $15^{\text {th }}$ October 2021

\footnotetext{
* Corresponding author: Dragana Jugović, Laboratory for Immunology and Genetics, Center for Medical and Clinical biochemistry, University Clinical Center Niš, Serbia. E-mail: dragana.jugovic@live.com
} 


\section{Introduction}

Colorectal cancer (CRC) is a malignant epithelial tumour of the colon which represents a health problem worldwide. It is the third most common cancer in men, the second in women, and the fourth most frequently diagnosed cancer in the world [1]. Until recently, it was thought that CRC occurs in older age, however, new studies suggest that this disease is increasingly diagnosed in the younger population, as well $[2,3]$.

Carcinogenesis of CRC is a gradual process where the clonal accumulation of genetic changes results in loss of control of the cell cycle [4]. Uncontrolled proliferation of mucosal epithelial cells results in polyps which can gradually grow even for 10 to 15 years before becoming cancerous [5]. The initial event in this process is the inactivation of the Adenomatous Polyposis Coli $(A P C)$ gene, which first triggers malignant mucosal transformation, while mutations in the Kirsten rat sarcoma viral oncogene homolog (KRAS) and TP53 genes later trigger the evolution of more aggressive subclones [6].

The KRAS gene encodes a small protein called KRAS that catalyzes the hydrolysis of guanosine triphosphate which means that it possesses GTPase activity. KRAS plays an important role in signal transduction cascades, such as growth factor receptor (EGFR)-activated signalling pathways, responsible for the control of multiple cellular functions including proliferation, motility, apoptosis, or survival $[7,8]$. In normal cells, KRAS proteins are activated after the binding of growth factors to tyrosine kinases receptors, changing their conformation from RAS GDP (inactive form) to RAS GTP (active form) [8]. Mutations in the KRAS gene occur early in the development of many cancers and often lead to the up-regulation of RAS proteins and their downstream effector pathways [8].

Having in mind that over expression of EGFR has been found in $80 \%$ of CRC and has been as- sociated with the progression of the malignant process and poor prognosis, it is no surprise that targeted therapy based on the application of monoclonal antibodies, such as cetuximab and panitumumab that inhibit EGFR signalling, has been shown to be very effective $[9,10]$. Namely, the action of these monoclonal antibodies is directed to the extracellular domain of EGFR where they block binding of a ligand, which results in a subsequent inhibition of the downstream RAS-RAF-MEK-ERK signalling pathway.

In Serbia, anti-EGFR therapy was registered in May 2009 as the third-line therapy for patients with metastatic colorectal cancer (mCRC) who had progression after therapy with irinotecan. However, although the use of targeted therapy with monoclonal antibodies has improved the survival of patients with CRC, it is indicated and effective only in a certain group of patients [11]. Namely, clinical trials have shown that carriers of the $K R A S$ gene mutation do not respond to the targeted EGFR therapy, which results in constitutive activation of the RAS-RAF-MEKERK pathway [12]. Because of that, wild-type (wt) status is pre necessary for the inclusion of this type of therapy, while patients with KRAS mutations are still being treated only by conventional methods such as surgical lesion removal, radiotherapy, and chemotherapy [13].

Despite the important role of general KRAS mutational status in the selection of the adequate therapeutic protocol in patients with CRC, studies that focus on its specific mutations and its significance on the progression of the disease are scarce.

The aim of this study was to determine the significance of specific KRAS mutations in response to standard chemotherapy protocols and to evaluate the correlation between these mutations and clinicopathological characteristics of CRC patients in Southeastern Serbia. 


\section{Materials and methods}

\section{Patient sample}

This study investigated the formalin-fixed paraffin-embedded (FFPE) sample tissues of 150 CRC patients who were referred to the Laboratory for Immunology and Genetics, The Center for Medical and Clinical biochemistry, University Clinical Center Niš, Serbia, for KRAS testing during 2015-2020. KRAS mutational status testing is required when the patient develops distant metastases after chemotherapy with oxaliplatin-based (FOLFOX 4, OXFL) in the first-line therapy and irinotecan-based chemotherapy (FOLFIRI, IFL) in the second-line one, which qualifies him/her as a potential candidate for anti-EGFR therapy. All procedures were carried out in concordance with the Declaration of Helsinki ethical guidelines and approved by the Ethics Committee of the Clinical Center of Niš, Serbia (Ethical Approval No 24722 /6).

All CRC patients were Caucasians. The following clinical and morphological data were collected for each patient: sex, age, tumour location, stage of tumour infiltration, nodal status and the presence of distant metastases. According to the location, the tumours were divided into the left-colon cancer (LCC) and right-colon cancer (RCC). LCC included splenic flexure, descending colon, sigmoid and rectum, while the RCC included caecum, ascending colon, hepatic flexure and transverse colon.

\section{DNA isolation}

Genomic DNA was extracted from the FFPE tumour tissue sections using a QIAamp DNA FFPE Tissue Kit (CE-IVD-marked; Qiagen, Hilden, Germany), according to the manufacturers' protocol. DNA quality was determined with $260 / 280$ optical density (OD) ratios in all samples and stored at $-20{ }^{\circ} \mathrm{C}$ until further use for KRAS genotyping. Genotyping was done by using two diagnostic methods: PCR amplification and hybridization and real-time PCR.

\section{PCR amplification and hybridization}

KRAS StripAssay ${ }^{\mathrm{TM}}$ (ViennaLab Diagnostics, Vienna, Austria) which detects the 10 most common mutations in codon 12 and13 (exon 2) was used for this method. Mutation detection was carried out following the manufacturer's instructions. Briefly, the total volume of each reaction sample was $25 \mu \mathrm{L}$ and consisted of $15 \mu \mathrm{L}$ of amplification mix, $5 \mu \mathrm{L}$ of diluted Taq DNA polymerase and $5 \mu \mathrm{L}$ of DNA template (concentaration: $5-8 \mathrm{ng} / \mu \mathrm{L})$.

Amplification was performed on iCycler Thermal Cycler (Bio-Rad, USA) starting with an initial denaturation step at $94^{\circ} \mathrm{C}$ for $2 \mathrm{~min}$, then running 35 cycles of $94^{\circ} \mathrm{C}$ for $60 \mathrm{~s}, 70^{\circ} \mathrm{C}$ for $50 \mathrm{~s}, 56^{\circ} \mathrm{C}$ for $50 \mathrm{~s}$ and $60^{\circ} \mathrm{C}$ for $60 \mathrm{~s}$, with a final extension at $60^{\circ} \mathrm{C}$ for $3 \mathrm{~min}$.

Following PCR, biotinylated amplification products were selectively hybridized to a test strip containing allele-specific oligonucleotide probes immobilized as an array of parallel lines, as shown in Fig. 1A, B, C.

Bound biotinylated sequences were detected using streptavidin-alkaline phosphatase and color substrates. For each polymorphic position, one of the two possible patterns was obtained -the presence or the absence of KRAS mutations hybridization bands (Fig. 1A, B, C).

\section{Real-time PCR}

The analysis of the KRAS mutation was performed using the "Easy® KRAS" Kit (Diatech Pharmacogenetics, Italy) with a Rotor-Gene 6000 - Corbett RT-PCR device (Diatech Pharmacogenetics, Italy), where list detectable mutations were: $K R A S$ codon $12-\mathrm{G} 12 \mathrm{R}(34 \mathrm{G}>\mathrm{C}), \mathrm{G} 12 \mathrm{~S}$ $(34 \mathrm{G}>\mathrm{A}), \mathrm{G} 12 \mathrm{C} \quad(34 \mathrm{G}>\mathrm{T}), \mathrm{G} 12 \mathrm{~A} \quad(35 \mathrm{G}>\mathrm{C})$, G12D (35G $>$ A), G12V (35G>T); KRAS codon 13 - G13D (38G>A); KRAS codon 59 (not distinguishable between them) - A59T (175G $>$ A), A59E $(176 \mathrm{C}>\mathrm{A})$, A59G $(176 \mathrm{C}>\mathrm{G}) ; K R A S$ codon 61 (not distinguishable between them) - Q61K (181C>A), Q61L (182A>T), Q61R 
A

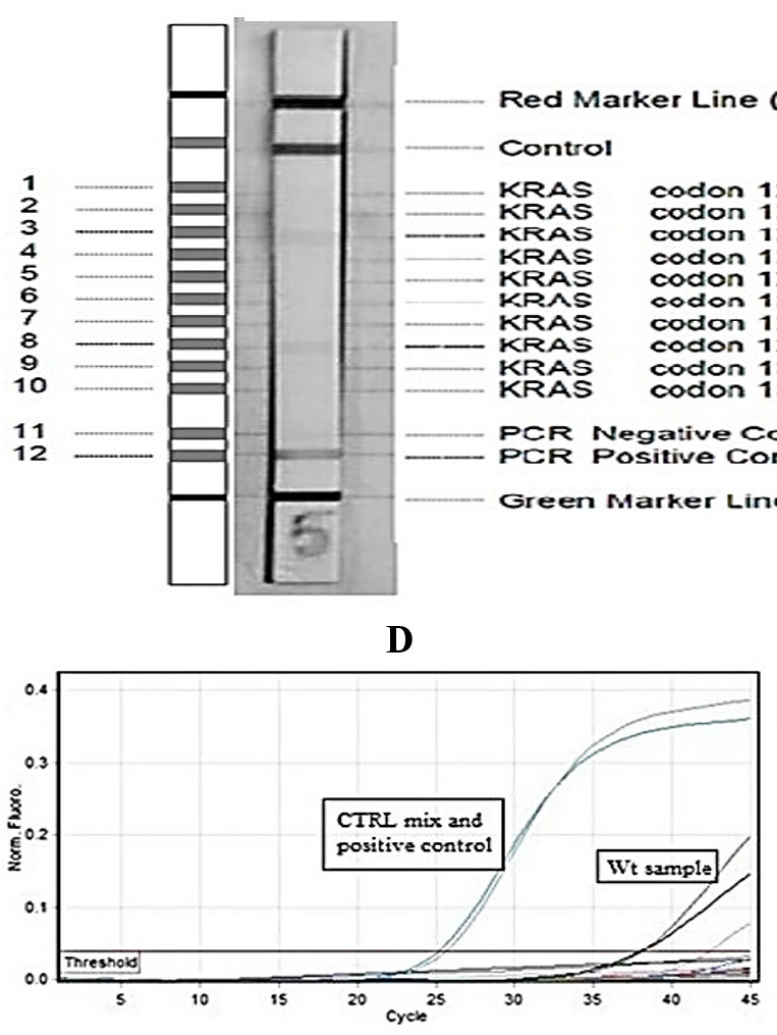

B

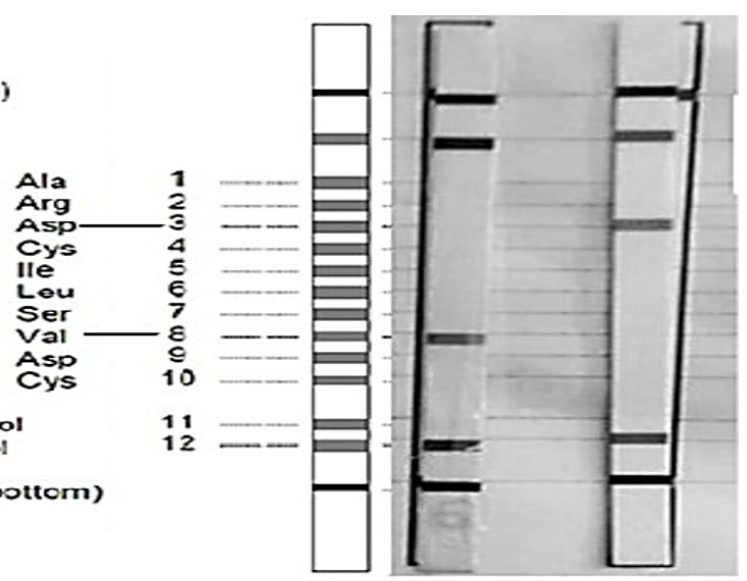

$\mathbf{E}$

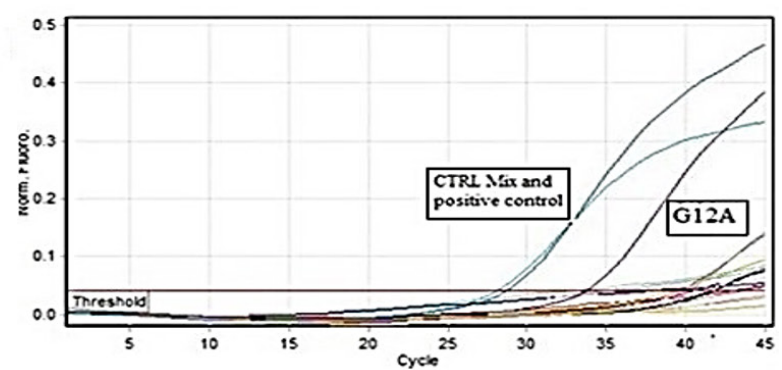

Fig. 1. The detection of $K R A S$ mutations with $K R A S$ Strip Assay: (A) no mutation (wt $K R A S$ sample), (B) G12V mutated sample, (C) G12D mutated sample (top side). Amplification plot detected by "Easy® KRAS" Kit: (D) no mutation (wt KRAS sample), (E) G12A mutated sample (bottom side).

$(182 \mathrm{~A}>\mathrm{G}), \mathrm{Q} 61 \mathrm{H}(183 \mathrm{~A}>\mathrm{C}), \mathrm{Q} 61 \mathrm{H}(183 \mathrm{~A}>\mathrm{T})$; $K R A S$ codon 117 (not distinguishable between them)- K117E(349A $>$ G), K117R $(350 \mathrm{~A}>\mathrm{G})$, $\mathrm{K} 117 \mathrm{~N}(351 \mathrm{~A}>\mathrm{T}), \mathrm{K} 117 \mathrm{~N}(351 \mathrm{~A}>\mathrm{C}) ; K R A S$ codon 146 (not distinguishable between them) - A146T (436G >A), A146P (436G>C), A146V $(437 \mathrm{C}>\mathrm{T})$.

$K R A S$ gene sequence was amplified using a mixture of $10 \mu \mathrm{L}$ Taq Premix, $4 \mu \mathrm{L}$ water, $1 \mu \mathrm{L}$ $K R A S$ ctrl mix(12) and either $5 \mu \mathrm{L}$ of the DNA sample or negative control or positive control. $K R A S$ gene sequence was amplified using the following cycling conditions initial denaturation step at $95^{\circ} \mathrm{C}$ for $2 \mathrm{~min}$, then running 40 cycles of $95^{\circ} \mathrm{C}$ for 10 seconds $/ 58^{\circ} \mathrm{C}$ for 60 seconds.

The mutation analysis was carried out in relation to the amplification of positive and negative control tests provided by the manufacturer and according to the included protocol.

\section{Statistical analyses}

The association between KRAS mutations and clinicopathological features of patients were determined using Fisher's exact test, with $\mathrm{P}<0.05$ considered statistically significant. Statistical analysis was performed using GraphPad Prism 5 (GraphPad Software, La Jolla California USA).

\section{Results}

\section{Clinicopathological characteristics of CRC patients}

In the present study, we analyzed colorectal tissue samples of 150 patients with histologically 
confirmed CRC. Male patients accounted for more than half of the cases $(n=103,68.6 \%)$. The age of patients ranged from 35 to 83 years old, where the mean age was 61 . There was a significantly higher frequency of LCC cancer $(n=113,75.3 \%)$ than RCC $(n=37,24.6 \%)$. Furthermore, tumours in the T3 stage of infiltration were more common $(n=102,68 \%)$ compared to T1/T2 and T4 stage. Clinico-pathological characteristics for 150 patients are summarized in Table 1.

\section{Distribution of KRAS mutations}

Of the total number of patients, KRAS mutations were present in 67 cases (45\%), while $83(55 \%)$ of the analyzed samples had wt $K R A S$ (Fig. 2A).

The most common mutations among the patients evaluated with KRAS mutations were found in codon $12(79.1 \%)$ and then in codon $13(10.4 \%)$. The glycine to aspartate in codon 12 (G12D) (Fig. 1C) was the most common mutation, accounting for $23(34.3 \%)$ of all the mutations identified. Mutation of glycine to valine (G12V) (Fig. 1B) was the second most common mutation with 16 cases $(23.8 \%)$. Mutation from glycine to alanine (G12A) (Fig. 1E) was identified in $11.9 \%$. Other identified mutations were mutation from glycine to aspartate on codon 13 (G13D) in codon Q61, glycine to cysteine $(\mathrm{G} 12 \mathrm{C})$ and glycine to serine (G12S) which constituted 10.4, 7.4, 5.9, and $2.9 \%$ of all cases, respectively. There was one
Table 1. Clinicopathological parameters of CRC patients enrolled in the study

\begin{tabular}{|c|c|c|c|}
\hline \multirow{2}{*}{\multicolumn{2}{|c|}{ Parameters }} & \multicolumn{2}{|c|}{ Total $(n=150)$} \\
\hline & & \multirow{2}{*}{$\begin{array}{c}\mathrm{n} \\
103\end{array}$} & \multirow{2}{*}{$\begin{array}{l}\% \\
68.6\end{array}$} \\
\hline \multirow{2}{*}{ Sex } & Male & & \\
\hline & Female & 47 & 31.4 \\
\hline \multirow{4}{*}{$\begin{array}{l}\text { Age at } \\
\text { diagnosis }\end{array}$} & $<50$ & 23 & 15.3 \\
\hline & $51-60$ & 32 & 21.3 \\
\hline & 61-70 & 70 & 46.6 \\
\hline & $>71$ & 25 & 16.6 \\
\hline \multirow{2}{*}{ Location } & Left-sided & 113 & 75.3 \\
\hline & Right-sided & 37 & 24.6 \\
\hline \multirow{3}{*}{$\begin{array}{l}\text { Tumor } \\
\text { infiltration }\end{array}$} & $\mathrm{T} 1 / \mathrm{T} 2$ & 21 & 14 \\
\hline & $\mathrm{T} 3$ & 102 & 68 \\
\hline & $\mathrm{T} 4$ & 27 & 18 \\
\hline \multirow{3}{*}{$\begin{array}{l}\text { Nodal } \\
\text { status }\end{array}$} & No & 48 & 32 \\
\hline & N1 & 59 & 39.3 \\
\hline & N2 & 43 & 28.6 \\
\hline \multirow{4}{*}{$\begin{array}{l}\text { Distant } \\
\text { metastasis }\end{array}$} & Liver & 79 & 52.6 \\
\hline & Lungs & 22 & 14.6 \\
\hline & Liver and lungs & 37 & 24.6 \\
\hline & Other & 12 & 8 \\
\hline
\end{tabular}

case with mutations in codon 117 , codon 59 and codon 146 (1.5\%), as shown in Fig. 2B.

\section{Clinicopathological characteristics of CRC patients in relation to the KRAS mutational status}

Based on the mutational status of KRAS there were no statistically significant differences in patients' gender, age, nodal status and distant
A

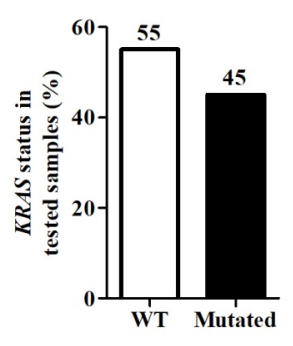

B

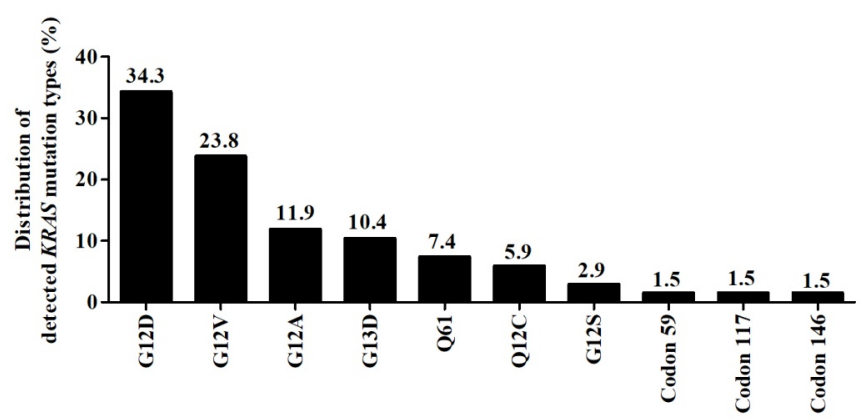

Fig. 2. (A) Frequency of wt $K R A S$ and mutated $K R A S$, (B) Distribution of detected $K R A S$ mutation types. 
metastases. However, a significant difference was found between RCC and LCC regarding the KRAS status, with KRAS mutation in RCC being 64.8\% (24/37), and in LCC 38\% (43/113) $(\mathrm{P}=0.0044)$. Moreover, the prevalence of the $K R A S$ mutations was significantly lower in the initial stages (T1/T2) than advanced tumour infiltration ( $\mathrm{T} 3$ and $\mathrm{T} 4$ stages) $(\mathrm{P}=0.0017)$, as shown in Table 2.

\section{Mutations in the KRAS gene and the time of appearance of distant metastases}

Most of the patients $(43.3 \%, 65 / 150)$ developed metastases in the first 6 months after the diagnosis, as shown in Table 3.

Since at the time of the testing for the presence of mutations in the KRAS gene all the patients included in this study, had already developed distant metastases, we divided them into two groups: those who developed metastases up to
6 months after diagnosis (poor therapeutic response) and those who developed metastases 24 months after diagnosis of the disease (good therapeutic response). The mutation rate of KRAS was different between these two groups of patients. KRAS mutations were significantly more common in patients who developed metastases up to 6 months after diagnosis than in the second group $(36.7 \%$ vs. $9.2 \%, \mathrm{P}=0.0083)$.

The most frequent KRAS mutations in a group of patients who developed metastasis during the first 6 months after the CRC diagnosis were in codons G12D and G12V (63.8\%), while the most prevalent mutation in patients with metastasis diagnosed after 24 months was in codon G12A (66.6\%), as shown in Fig. 3.

\section{Discussion}

In Serbia, CRC is the second most common carcinoma, as well as the second most common cause

Table 2. Correlation between $K R A S$ mutation status and clinicopathological parameters of CRC patients.

\begin{tabular}{|c|c|c|c|c|c|c|}
\hline \multirow{3}{*}{ Parameter } & & \multicolumn{4}{|c|}{ KRAS status $(n=150)$} & \multirow{3}{*}{ P value* } \\
\hline & & \multicolumn{2}{|c|}{ Mutated } & \multicolumn{2}{|c|}{ WT } & \\
\hline & & $\mathrm{n}$ & $\%$ & $\mathrm{n}$ & $\%$ & \\
\hline \multirow{2}{*}{ Sex } & Male & 43 & 41.7 & 60 & 58.3 & \multirow{2}{*}{0.287} \\
\hline & Female & 24 & 51 & 23 & 49 & \\
\hline \multirow{4}{*}{ Age at diagnosis } & $<50$ & 11 & 47.8 & 12 & 52.2 & \multirow{4}{*}{0.9442} \\
\hline & $51-60$ & 15 & 46.9 & 17 & 53.1 & \\
\hline & $61-70$ & 31 & 44.3 & 39 & 55.7 & \\
\hline & $>71$ & 10 & 40 & 15 & 60 & \\
\hline \multirow{2}{*}{ Location } & Left-sided & 43 & 38 & 70 & 62 & \multirow{2}{*}{0.0044} \\
\hline & Right-sided & 24 & 64.8 & 13 & 35.2 & \\
\hline \multirow{3}{*}{ Tumor infiltration } & $\mathrm{T} 1 / \mathrm{T} 2$ & 2 & 9.5 & 19 & 90.5 & \multirow{3}{*}{0.0017} \\
\hline & T3 & 53 & 52 & 49 & 48 & \\
\hline & $\mathrm{T} 4$ & 12 & 44.4 & 15 & 55.5 & \\
\hline \multirow{3}{*}{ Nodal status } & No & 19 & 39.6 & 29 & 60.4 & \multirow{3}{*}{0.6883} \\
\hline & N1 & 28 & 47.5 & 31 & 52.5 & \\
\hline & N2 & 20 & 46.5 & 23 & 53.5 & \\
\hline \multirow{4}{*}{ Distant metastasis } & Liver & 38 & 48.1 & 41 & 51.9 & \multirow{4}{*}{0.0555} \\
\hline & Lungs & 9 & 40.1 & 13 & 59.9 & \\
\hline & Liver and lungs & 19 & 51.3 & 18 & 48.7 & \\
\hline & Other & 1 & 8.3 & 11 & 91.7 & \\
\hline
\end{tabular}

*Fisher's exact test, $\mathrm{P}<0.05$ statistically significant (bolded) 
Table 3. Association of $K R A S$ mutations types with time from diagnosis of CRC to the development of metastases.

\begin{tabular}{cccccc}
\hline \multirow{2}{*}{ Time after CRC diagnosis (months) } & \multicolumn{2}{c}{ Mutated $\boldsymbol{K R} \boldsymbol{A} \boldsymbol{S}$} & \multicolumn{2}{c}{ WT $\boldsymbol{K R} \boldsymbol{A} \boldsymbol{S}$} & \multirow{2}{*}{ P value* } \\
\cline { 2 - 5 } & $\mathbf{n}$ & $\mathbf{\%}$ & $\mathbf{n}$ & $\mathbf{\%}$ & \\
\hline$<6$ & 36 & 36.7 & 29 & 29.5 & \multirow{2}{*}{$\mathbf{0 . 0 0 8 3}$} \\
\hline $24 \leq$ & 9 & 9.2 & 24 & 24.5 & \\
\hline
\end{tabular}

*Fisher's exact test, $\mathrm{P}<0.05$ statistically significant (bolded)

of cancer-related death, right after lung cancer in men, and breast cancer in women [14]. In addition to that fact, recent data show that the number of cases of CRC in Serbia was found to be on the $4 \%$ rise between the years 2014 and 2017 [15]. $\mathrm{CRC}$ is a very heterogeneous disease both in terms of its pathogenesis and in terms of molecular genetic characteristics [16]. To date, more than 3,000 KRAS mutation sites with different biological effects have been reported [17]. Although $K R A S$ mutational status is known to be a selective marker for predicting responses to monoclonal antibodies against EGFR, its precise prognostic value remains controversial [18]. Therefore, precise identification and characterization of mutational biomarkers with therapeutic and/or prognostic value will be of crucial importance for a personalized approach to people with CRC.

The results of this study showed that $45 \%$ $(67 / 150)$ of tested CRC patients had mutations in the KRAS gene, and the rest of them $(55 \%)$ had wt KRAS (Fig. 2A), which is in concordance with previous studies [19,20,21]. Interestingly, compared to the European and Latin American population, the frequency of $K R A S$ mutations occurrence is much lower in Asian patients (24\%) [22]. This difference can be the result of many factors such as environmental and lifestyle conditions or epigenetic differences between those ethnic groups.

The most frequently observed KRAS mutations in our study were located in codon $12(79.1 \%)$ and codon 13 (10.4\%) of exon 2 (Fig. 2B) which agrees with a previous study which found that among all detected mutations in KRAS more than $70 \%$ are located in codon 12 [23].

Among the mutations examined in $K R A S$, the mutation rate of G12D was the highest (34.3\%) followed by G12V (23.8\%). Additionally, similar to the results of other authors [24,25] we noticed that mutations G12D and G12V are the most prevalent in the Serbian population.
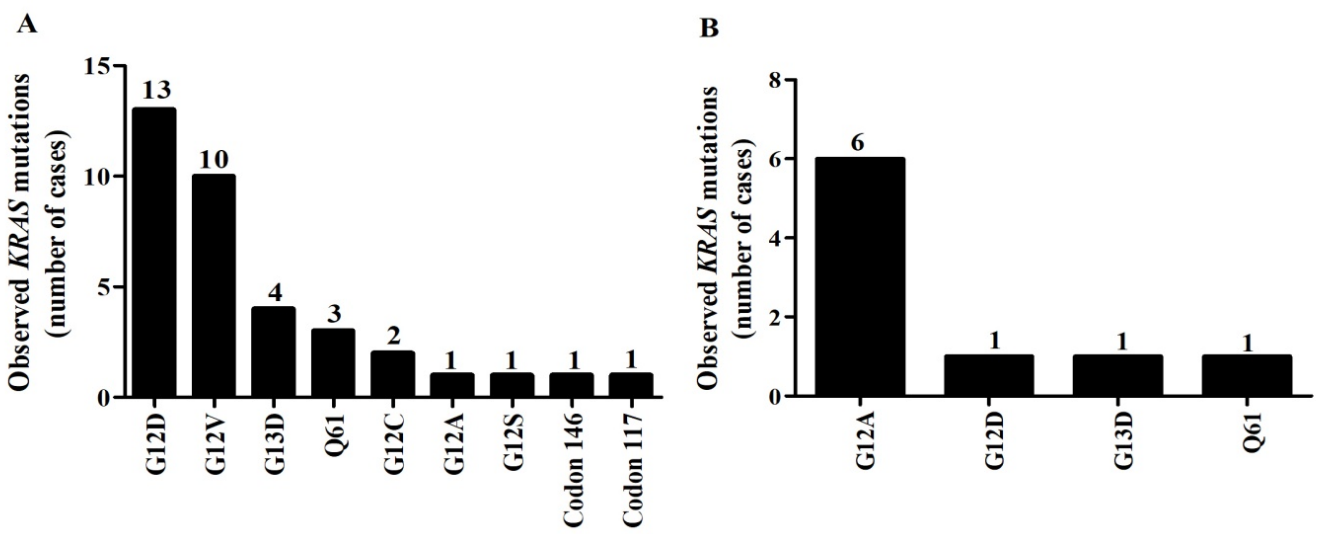

Fig. 3. Prevalence of $K R A S$ mutations after the diagnosis of CRC. (A) in patients who developed metastasis after 6 months, (B) in patients who developed metastasis after 24 months. 
We found that the frequency of mutations in codons 59, 117 and 146 were $1.5 \%$, which is in concordance with the study of Awidi et al. [26] while a higher prevalence of the mutation in codon 146 was observed in patients from Thailand, where $8.3 \%$ of them had this mutation [27].

Many authors evaluated correlations between mutations in $K R A S$, age and sex of patients with clinicopathological characteristics of CRC and some of the results indicate that both tumor location and $K R A S$ status play important roles in the prognosis of CRC patients $[19,20,28]$. Patients with LCC and mutated $K R A S$ have a poorer prognosis compared with wt $K R A S$, but RCC patients with $K R A S$ mutation has no significant difference in overall survival rate compared with patients with wt KRAS [28]. Also, patients with RCC have a worse prognosis and higher mortality compared to patients with LCC [29]. Our data indicate that the KRAS mutation is much more common in the RCC than in the LCC (64.8\% vs. $38 \% ; \mathrm{P}=0.0044$ ) (Table 2) which is in favour of being associated with a poorer prognosis of the disease. The study of Baran et al. suggests that the response to therapy is also completely different between these tumour entities, with LCC patients benefiting more from adjuvant therapy [30]. These differences can be explained by the fact that the left and right colon have a different embryonic origin, which may result in different molecular carcinogenic characters including $K R A S$ mutations [28].

In our study, all subjects received oxaliplatin-based chemotherapy (FOLFOX 4, OXFL) as first-line therapy. As the second-line one, they received irinotecan-based chemotherapy (FOLFIRI, IFL), and by the time they were tested for $K R A S$ gene mutations, they had already developed the distant metastases.

One of the goals of our work was to determine the significance of the mutational status of the $K R A S$ gene as a possible predictive biomarker of response to applied chemotherapeutic protocols.
Our results showed that almost half of the patients included in the study developed metastasis in the first 6 months since the diagnosis of CRC (65/150, 43.3\%), while 22\% (33/150) developed metastasis 24 months after the diagnosis. When we compared the presence of KRAS gene mutations only in patients who developed metastases after 6 months and after 24 months, we noticed that the presence of the mutation was significantly more common in the first group of patients (36.7\% vs. $9.2 \%, \mathrm{P}=0.0083$ ).

Here, we provide additional evidence on the impact of $K R A S$ mutations on the progression of colorectal carcinoma, which was confirmed recently by different authors $[19,31]$.

Although the prognostic value of specific point mutations has not yet been fully clarified [32] this study demonstrates that in the group of patients where metastases appeared in up to 6 months, the most common specific mutations were G12D and G12V accounting together for $63.88 \%(23 / 36)$. These results associate the G12D and G12V mutations with poor therapeutic response in $\mathrm{CRC}$ patients who received oxaliplatin-based chemotherapy (FOLFOX 4, OXFL) as the first-line therapy and irinotecan-based chemotherapy (FOLFIRI, IFL) as the second-line therapy. Several studies explained that the aggressive behaviour of the G12D and G12V mutation is due to the production of proteins that behave differently than other mutated KRAS proteins, with very high transforming potential and a low GTP intrinsic and GAP-mediated GTP hydrolysis [33,34].

We also found that in the group of patients where metastases appeared after 24 months, mutation G12A was the most prevalent $66.66 \%$ (6/9) and also associated with good therapeutic response in patients with CRC which received oxaliplatin-based chemotherapy FOLFOX 4 and irinotecan-based chemotherapy FOLFIRI. In contrast to our data, the Fiala et al. study reported that G12A KRAS mutation is associated with poor therapeutic response in patients with $\mathrm{CRC}$ receiving bevacizumab [35]. Since none of our 
patients received bevacizumab, our findings reveal new possibility for further investigations in order to determine the connection of mutational status, especially G12A KRAS mutation, and response to different therapeutic protocols.

\section{Conclusion}

The prevalence of $K R A S$ gene mutations in CRC patients from Southern Serbia was relatively high. In a group of patients with a poor therapeutic response to oxaliplatin-based chemotherapy (FOLFOX 4, OXFL) as first-line therapy and irinotecan-based chemotherapy (FOLFIRI, IFL) as second line therapy, the most frequent mutations found in KRAS gene were G12D and D12V. In a group of patients with a good response to the aforementioned therapeutic protocols, the most prevalent mutation was G12A. Also, we showed that tumour location and specific KRAS mutational status may be a significant prognostic factor. However, the results of our study indicate that there is a significant difference in biological behaviour between tumours harbouring different mutations in the KRAS gene and they also open new possibilities for further investigations in order to determine predictive biomarkers of patient response to different therapeutic protocols.

\author{
Abbreviations \\ CRC - colorectal cancer \\ APC - Adenomatous Polyposis Coli \\ KRAS - kirsten rat sarcoma viral oncogene ho- \\ molog \\ EGFR-epidermal Growth Factor Receptor \\ Wt- wild-type \\ PCR-polymerase chain reaction \\ LCC- left colon cancer \\ $\mathrm{RCC}$ - right colon cancer
}

\section{Authors' contribution}

D. J. - participated in designing and performing study; writing the original draft;
M. V. N.- analyzed the data, manuscript preparation;

V. M. - DNA isolation, formal analysis, critical revision of the manuscript;

LJ. B.- experiments performing, DNA isolation;

R. M.- experiments performing, DNA isolation;

G. S.- collected samples, formal analysis;

P. V.- analyzed the data, manuscript preparation. All authors have read and approved the final manuscript all authors agree to be accountable for all aspects of the work.

\section{Acknowledgments}

This study was not financially supported.

\section{Conflict of Interest Statement}

The authors have no conflicts of interest to declare.

\section{References}

1. Rawla P, Sunkara T, Barsouk A. Epidemiology of colorectal cancer: incidence, mortality, survival, and risk factors. Prz Gastroenterol. 2019;14(2):89-103. DOI: 10.5114/pg.2018.81072

2. Campos FG. Colorectal cancer in young adults: A difficult challenge. World J Gastroenterol. 2017;23(28):5041-5044. DOI: 10.3748/wjg.v23. i28.5041

3. Connell LC, Mota JM, Braghiroli MI, Hoff PM. The Rising Incidence of Younger Patients With Colorectal Cancer: Questions About Screening, Biology, and Treatment. Curr Treat Options Oncol. 2017;18(4):23. DOI: $10.1007 / \mathrm{s} 11864-017-0463-3$

4. Mármol I, Sánchez-de-Diego C, Pradilla DA, Cerrada E, Rodriguez Yoldi MJ. Colorectal carcinoma: A general overview and future perspectives in colorectal cancer. Int J Mol Sci. 2017;18(1):197. DOI: 10.3390/ ijms 18010197

5. Recio-Boiles A, Cagir B. Colon Cancer. [Updated 2021 Jan 25]. In: StatPearls [Internet]. Treasure Island (FL): StatPearls Publishing; 2021 Jan-. Available from: https:/www.ncbi.nlm.nih.gov/books/NBK470380/

6. Maffeis V, NicolèL, Cappellesso R. RAS, cellular plasticity, and tumor budding in colorectal cancer. FronOncol. 2019;9;1255. DOI: 10.3389/fonc.2019.01255

7. Buday L, Downward J. Many faces of Ras activation. Biochimica et Biophysica Acta. 2008;1786(2):178-87. 
DOI: 10.1016/j.bbcan.2008.05.001

8. Takács T, Kudlik G, Kurilla A, Szeder B, Buday L, Vas V. The effects of mutant Ras proteins on the cell signalome. Cancer Metastasis Rev. 2020;39:1051-65. DOI: $10.1007 / \mathrm{s} 10555-020-09912-8$

9. Hwang K, Yoon JH, Lee JH, Lee S. Recent Advances in Monoclonal Antibody Therapy for Colorectal Cancers Biomedicines. 2021; 9(1):39. DOI: 10.3390/biomedicines 9010039

10. Porebska I, Harlozińska A, Bojarowski T. Expression of the tyrosine kinase activity growth factor receptors (EGFR, ERB B2, ERB B3) in colorectal adenocarcinomas and adenomas. Tumour Biol. 2000;21(2):105-15. DOI: $10.1159 / 000030116$

11. Dassonville O, Bozec A, Fischel JL, Milano G. EGFR targeting therapies: Monoclonal antibodies versus tyrosine kinase inhibitors. Similarities and differences. Crit Rev Oncol Hematol. 2007;62:53-61. DOI: 10.1016/j. critrevonc.2006.12.008

12. Porru M, Pompili L, Caruso C, Biroccio A, Leonetti C. Targeting KRAS in metastatic colorectal cancer: current strategies and emerging opportunities. J Exp Clin Cancer Res. 2018;37(1):57. DOI: 10.1186/s13046-0180719-1

13. De Roock W, Jonker DJ, Di Nicolantonio F, Sartore-Bianchi A, Tu D, Siena S, et al. Association of KRAS p.G13D mutation with outcome in patients with chemotherapy-refractory metastatic colorectal cancer treated with cetuximab. JAMA 2010;304(16):1812-20. DOI: 10.1001/jama.2010.1535

14. BankovicLazarević D, Krivokapić Z, Barišić G, Jovanović V, Ilić D, Veljković M. Organized colorectal cancer screening in Serbia - the first round within 20132014. Vojnosanit Pregl. 2016;73:360-7. DOI: 10.2298/ VSP150421113B

15. Vekic B, Dragojevic-Simic V, Jakovljevic M, Pilipovic F, Simic R, Zivic R, et al. Medical cost of colorectal cancer services in Serbia between 2014 and 2017: National data report. Front Pharmacol. 2019;10:526. DOI: 10.3389/fphar.2019.00526

16. Patel A, Tripathi G, Gopalakrishnan K, Williams N, Arasaradnam RP. Field cancerisation in colorectal cancer: a new frontier or pastures past? World J Gastroenterol. 2015;21(13):3763-72. DOI: 10.3748/wjg.v21. i13.3763

17. Lea IA, Jackson MA, Li X, Bailey S, Peddada SD and Dunnick JK. Genetic pathways and mutation profiles of human cancers: site- and exposure-specific patterns. Carcinogenesis. 2007;28:1851-8. DOI: 10.1093/carcin/ bgm 176

18. Sorich MJ, Wiese MD, Rowland A, Kichenadasse G, McKinnon RA, Karapetis CS. Extended RAS mutations and anti-EGFR monoclonal antibody survival benefit in metastatic colorectal cancer: A meta-analysis of randomized, controlled trials. Ann Oncol. 2015;26:13-21.
DOI: $10.1093 / \mathrm{annonc} / \mathrm{mdu} 378$

19. Garcia-Carbonero N, Martinez-Useros J, Li W, Orta A, Perez N, Carames C, et al. KRAS and BRAF mutations as prognostic and predictive biomarkers for standard chemotherapy response in metastatic colorectal cancer: A single institutional study. Cells 2020;9(1):219. DOI: 10.3390/cells9010219

20. Bruera G, Cannita K, Di Giacomo D, Lamy A, FrébourgT, Sabourin JS, et al. Worse prognosis of KRASc.35 G > A mutant metastatic colorectal cancer (MCRC) patients treated with intensive triplet chemotherapy plus bevacizumab (FIr-B/FOx). BMC Med. 2013;11:59. DOI: 10.1186/1741-7015-11-59

21. Jones RP, Sutton PA, Evans JP, Clifford R, McAvoy A, Lewis J, et al. Specific mutations in KRAS codon 12 are associated with worse overall survival in patients with advanced and recurrent colorectal cancer. $\mathrm{Br} \mathrm{J}$ Cancer. 2017;116(7):923-9. DOI: 10.1038/bjc.2017.37

22. Sirisena ND, Deen K, Mandawala DEN, Herath P, Dissanayake VHW. The pattern of KRAS mutations in metastatic colorectal cancer: a retrospective audit from Sri Lanka. BMC Research Notes. 2017;10(1):392. DOI: 10.1186/s13104-017-2731-5

23. SadoughA, Afshari M, Rostami F, Berzegari S, Janbabaee $\mathrm{G}$, Tabrizi R, et al. A systematic review and meta-analysis on the prevalence of KRAS gene mutation in samples of colorectal cancer. WCRJ. 2020;7:e1522.

24. Jakovljevic K, Malisic E, Cavic M, Krivokuca A, Dobricic J, Jankovic R. KRAS and BRAF mutations in Serbian patients with colorectal cancer. J BUON. 2012;17(3):575-80.

25. Jankovic R, Brotto K, MalisicE,Krivokuca A, Cavic M, Jovanovic K, Boljevic I, Tanic M, Radulovic S. KRAS mutations in colorectal cancer patients in Serbia: 5 years experience of central testing. Ann Oncol. 2014;25 (Supplement 2): ii14-ii104.

26. Awidi M, Ababneh N, Shomaf M, AlFararjehF,Owaidi $\mathrm{L}, \mathrm{Al}$ Khatib $\mathrm{M}$, et al. KRAS and NRAS mutational gene profile of metastatic colorectal cancer patients in Jordan. PloS One. 2019;14(12):e0226473. DOI: 10.1371/journal.pone.0226473

27. Korphaisarn K, Pongpaibul A, Roothumnong E,Pongsuktavorn K , Thamlikitkul L, Anekpuritanang T, et al. High frequency of KRAS codon 146 and FBXW7 mutations in Thai patients with stage II-III colon cancer. Asian Pac J CancerPrev. 2019;20(8):2319-26. DOI: 10.31557/APJCP.2019.20.8.2319

28. Xie MZ, Li JL, Cai ZM, Li KZ, Hu BL. Impact of primary colorectal cancer location on the KRAS status and its prognostic value. BMC Gastroenterol. 2019;19(1):46. DOI: 10.1186/s12876-019-0965-5

29. Bylsma LC, Gillezeau C, GarawinTA,Kelsh MA, Fryzek JP, Sangare L, Lowe KA.Prevalence of RAS and BRAF mutations in metastatic colorectal cancer pa- 
tients by tumor sidedness: A systematic review and meta-analysis. Cancer Med. 2020;9(3):1044-57. DOI: 10.1002/cam4.2747

30. Baran B, OzupekNM, Tetik NY, Acar E, Bekcioglu O, Baskin Y. Difference between left-sided and right-sided colorectal cancer: a focused review of literature. Gastroenterology Res. 2018;11:264-73. DOI: 10.14740/ gr1062w

31. Dai D, Wang Y, Zhu L, Jin H, Wang X. Prognostic value of KRAS mutation status in colorectal cancer patients: a population-based competing risk analysis. Peer J. 2020;8:e9149. DOI: 10.7717/peerj.9149

32. Li W, Qiu T, Zhi W, Shi S, Zou S, Ling Y, Shan L, Ying J, Lu N. Colorectal carcinomas with KRAS codon 12 mutation are associated with more advanced tumor stages BMC Cancer. 2015;15:340. DOI: 10.1186/ s12885-015-1345-3
33. Hunter JC, Manandhar A, Carrasco MA, Gurbani D, Gondi S, Westover KD. Biochemical and structural analysis of common cancer-associated KRAS mutations. MolCancer Res. 2015;13:1325-35. DOI: 10.1158/1541-7786.MCR-15-0203

34. Mu-oz-Maldonado C, Zimmer Y, Medová M. A Comparative Analysis of Individual RAS Mutations in Cancer Biology. Front Oncol. 2019;18;9:1088. DOI: 10.3389/fonc. 2019.01088

35. Fiala O, Buchler T, Mohelnikova-Duchonova B, Melichar B, Matejka VM, Holubec L, Kulhankova J, Bortlicek Z, Bartouskova M, Liska V, Topolcan O, Sedivcova M, Finek J. G12V and G12A KRAS mutations are associated with poor outcome in patients with metastatic colorectal cancer treated with bevacizumab. Tumour Biol. 2016;37(5):6823-30. DOI: 10.1007/s13277015-4523-7 